\title{
A Modelling Framework for Characterising the Impacts of Uncertainty on Energy Systems
}

\author{
Yongning Zhao, Meysam Qadrdan, Nick Jenkins \\ School of Engineering, Cardiff University, Cardiff, UK \\ \{zhaoy88, qadrdanm, jenkinsn6\}@ cardiff.ac.uk
}

\begin{abstract}
A modelling framework was developed for characterising the impacts of variability and uncertainty of renewable generation and load on a multi-vector and multi-scale energy system. A time series synthesis algorithm was proposed to produce a large number of daily profiles for wind, PV and load, representing possible variation features. Based on the synthetic time series, four models with different uncertainty characteristics were built and applied to generate forecast scenarios for wind and PV. Using the generated scenarios, the operation of a combined gas and electricity system was formulated as a two-stage stochastic mixedinteger linear optimisation problem. A simplified Great Britain's energy system was investigated under different flexibility options and uncertainty characterisations. Results indicate that electricity storage was the most effective measure to reduce operation cost. The impacts of uncertainty characterisation methods were significant only when system flexibility was insufficient.
\end{abstract}

Index Terms-integrated energy system, multi-scale, optimisation, variability, uncertainty

\section{INTRODUCTION}

The energy system in the UK is undergoing radical changes to achieve the renewable targets as well as the newly proposed net-zero goal [1]. A range of future scenarios have been envisaged to describe possible pathways to a low carbon energy system [2]. These scenarios are characterised by high integration levels of renewable energy and electrification. Therefore, the variability and uncertainty from renewable energy supply and demand will pose challenges to the safe and economic operation of power systems [3].

Meanwhile, it is widely recognised that these challenges can be effectively handled through coupling between power systems and other energy sectors (e.g. gas and heat) which can provide sufficient flexibility (i.e. the capability of a system to allocate its resources to compensate its net load variability) to support power system operation [4]. Flexibility technologies vary depending on sectors and scales [5], including line-pack of gas pipelines [6], batteries [7], demand response [8], etc .

Different optimisation models have been proposed to exploit flexibility under uncertainty [9]. Among them, stochastic optimisation [10] and robust optimisation [11] are most typical. To design appropriate optimisation models making the most of the flexibility from an integrated energy system, it is essential to understand the impact of variability and uncertainty on the operation of electricity systems and the efficacy of novel

This work was supported by the UK Engineering and Physical Sciences Research Council (EPSRC) grant EP/S001492/1 for project entitled MaxImiSing flexibility through multi-Scale IntegratiON of energy systems (MISSION). strategies and flexible technologies to mitigate the impacts [3]. The impacts of variable generation and transmission constraints on power system flexibility were assessed [12] [13]. In [14], the impact of large-scale wind integration on the operation of integrated electricity and gas system in Great Britain (GB) was studied. The impact of spatio-temporal and inter-annual variability of weather was studied to design lowcarbon power systems for GB in 2050 [15]. However, previous studies were in a deterministic optimisation framework or didn't consider multiple scales of a energy system. Therefore, it is important to investigate how different sectors at different scales interact within the stochastic optimisation framework by using different uncertainty characterisation models.

The contributions of this paper are: 1) A complete modelling framework was presented to investigate the impact of variability and uncertainty on the future energy system operation; 2) A method for variability characterisation and time series synthesis was proposed to generate a large number of daily profiles for uncertainty sources; 3 ) Different uncertainty characterisation models were applied to generate forecast scenarios for a two-stage stochastic dispatch optimisation; 4) The framework was exemplified by a simplified representation of a GB multi-vector and multi-scale (MVMS) energy system, considering available flexibility for different scales and sectors (e.g. gas-fired generation, storage, demand response).

\section{MODELLING FRAMEWORK}

Variability sources considered include wind, PV and electrical load at different scales. In addition, the wind and PV also have forecasting uncertainties. The complete framework is illustrated in Fig. 1, which includes the following procedures.

1) Specific probability distributions were created for variability sources at different scales using historical data.

2) A low carbon scenario representing a plausible future of the GB energy system was defined.

3) A large number of daily profiles with hourly resolution were produced, based on the probability distributions and temporal characteristics.

4) For each daily profile of wind and PV power, forecast scenarios were generated by using four different uncertainty characterisation models.

5) A two-stage stochastic optimisation model was developed for a simplified representation of the GB energy system in 2030. Selected results were used to analyse the impacts of variability and uncertainty. 


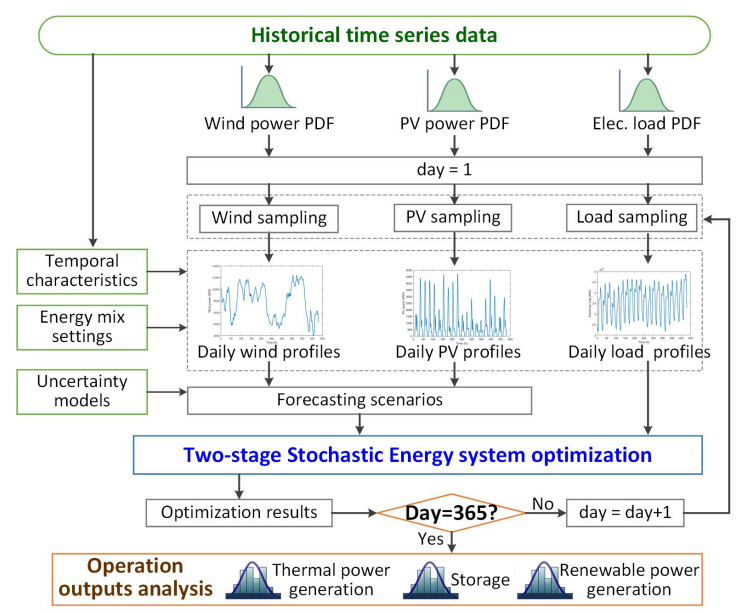

Fig. 1. The framework of the study

\section{VARIABILITY AND UNCERTAINTY CHARACTERISATION}

\section{A. Variability characterisation of wind and $P V$}

Wind and PV power generation are stochastic and intermittent and are driven by natural characteristics.

Normalised historical wind power data was transformed to Gaussian data to deal with the double-bounded feature of wind power [16]. Then a Gaussian distribution model $Y^{\prime}\left(y^{\prime} ; \mu, \sigma\right) \sim \mathcal{N}\left(\mu, \sigma^{2}\right)$ was built to generate samples $y^{\prime}$ which were transformed back to normalised wind power $x_{w}$ using the inverse Logit transformation. The Beta distribution function [17] was used to model the PV power generation during daytime, i.e., $X\left(x_{p v} ; \alpha, \beta\right) \sim \operatorname{Be}(\alpha, \beta)$.

In addition to the probability distributions, a novel adaptive Markov chain approximation (AMCA) was used to retain the intrinsic temporal characteristics during the random sampling procedure. Firstly, the Markov state transition probability matrix was trained by historical data and expressed by:

$$
\boldsymbol{\Lambda}=\left[\begin{array}{llll}
\boldsymbol{\lambda}_{1} & \boldsymbol{\lambda}_{2} & \ldots & \boldsymbol{\lambda}_{n}
\end{array}\right]^{\top}=\left(\lambda_{a b}\right)_{(a, b=1,2, \ldots, n)}
$$

where $n$ is the total number of equally partitioned states of historical normalised data, $\lambda_{a b}$ is the probability of transition from state $a$ to state $b$. To force the random samples to follow the matrix $\boldsymbol{\Lambda}$, an adaptive approximation transition matrix $\Psi^{(t)}$ initialised with zeros was set for the generated samples:

$$
\boldsymbol{\Psi}^{(t)}=\left[\begin{array}{llll}
\boldsymbol{\psi}_{1} & \boldsymbol{\psi}_{2} & \ldots & \boldsymbol{\psi}_{n}
\end{array}\right]^{\top}=\left(\psi_{a b}^{(t)}\right)_{(a, b=1,2, \ldots, n)}
$$

The matrix $\Psi^{(t)}$ was updated at each time $t$ by the new sample and then was used along with the matrix $\boldsymbol{\Lambda}$ to generate the next sample. The $\boldsymbol{\Psi}^{(t)}$ asymptotically approximates to the $\Lambda$ with increasing number of generated samples. The detailed rules of AMCA are given in Algorithm 1.

According to the diurnal pattern of PV power, samples for night time were set to zero, otherwise the Beta distribution and AMCA were used to generate samples. Additionally, two separate Markov chain models were trained for ramp-up in the morning and ramp-down in the afternoon.

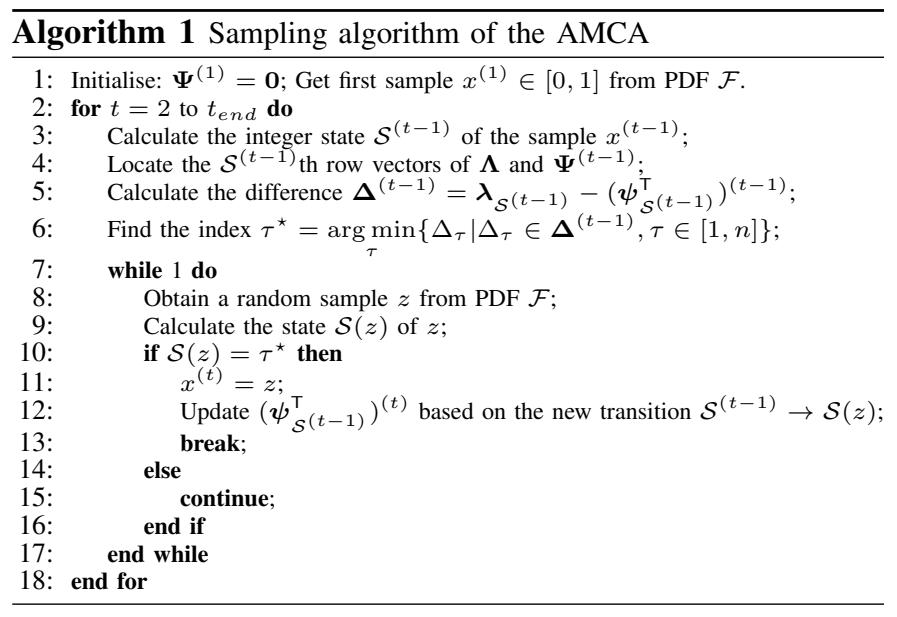

Furthermore, to account for seasonal patterns of wind power and PV power [18] [19], the following models were applied.

$$
\begin{aligned}
& x_{w}^{\prime}=(1-2 \epsilon) x_{w}+\epsilon(\cos (2 \pi t / 8760)+1) \\
& x_{p v}^{\prime}=(1-2 \epsilon) x_{p v}+\epsilon(\sin (2 \pi t / 8760-\pi / 2)+1)
\end{aligned}
$$

where $\epsilon$ is a very small positive constant number (e.g., 0.05).

Finally the normalised sample data in the interval $[0,1]$ were converted to values according to their assumed capacity.

\section{B. Variability characterisation of electrical load}

The electrical load profiles have strong daily, weekly and seasonal patterns. To generate load profiles, a standard daily load profile was obtained by averaging all daily load profiles of a historical year. Random load samples were generated from the Gamma distribution function [20]. The samples were retained if they were within a predefined deviation from the standard profile and followed the varying trend of the standard profile. The seasonal effect was modelled as

$$
x_{l}^{\prime}=(1-2 \epsilon) x_{l}+\epsilon(\cos (2 \pi t / 8760)+1),
$$

If a sample was within weekends, it was down by a ratio, e.g. 0.8. The samples were finally scaled by peak load.

\section{Uncertainty characterisation}

Using the generated data, forecasts and forecast errors were obtained by Extreme Learning Machine [21] for wind power and PV power for each time horizon of 24 hours. The method in [22] was used to generate forecast scenarios.

Four uncertainty characterisation models were considered, i.e., Multivariate Empirical (ME) Distribution and Multivariate Normal (MN) Distribution considering temporal correlation of errors at different horizons, and Univariate Empirical (UE) Distribution and Univariate Normal (UN) Distribution assuming no correlation. For the $\mathrm{ME}$ and $\mathrm{MN}$, the Pearson correlation matrix of errors was used as co-variance matrix.

1000 daily scenarios of wind power and PV power were generated for each of 365 days. The number of scenarios for each source was reduced to 4 using a clustering method. Note that the actual number of scenarios for each day was $4^{4}=256$ by combining the scenarios of 4 different uncertainty sources, i.e., wind and PV at different scales. 


\section{OPERATION MODEL OF ENERGY SYSTEM}

The operation model was formulated as a two-stage stochastic optimisation problem which takes forecast scenarios as inputs. The scheduling horizon is 24 hours with hourly time resolution. The model was run for 365 consecutive days. The energy system model doesn't consider network constraints.

\section{A. Objective Function}

The two-stage least-cost objective function [10] [23] is :

$$
\begin{aligned}
& \min T C=\sum_{t=1}^{T}\left\{\sum_{g=1}^{N_{E}}\left(C_{g}^{E} P_{g, t}+C_{g}^{C U} R_{g, t}^{U}+C_{g}^{C D} R_{g, t}^{D}\right)\right. \\
& \left.+C^{I M} P_{t}^{I M}+\sum_{g t=1}^{N_{G}} C^{G} Q_{g t, t}+\sum_{g s=1}^{N_{G S}}\left(C^{I} Q_{g s, t}^{I}+C^{O} Q_{g s, t}^{O}\right)\right\} \\
& +\sum_{m=1}^{N_{M}} \pi_{m}\left\{\sum _ { t = 1 } ^ { T } \left\{\sum_{g=1}^{N_{E}}\left(C_{g}^{U R} r_{g, t, m}^{U}-C_{g}^{D R} r_{g, t, m}^{D}\right)\right.\right. \\
& +\sum_{g s=1}^{N_{G S}}\left(C^{O u} Q_{g s, t, m}^{O u}+C^{I u} Q_{g s, t, m}^{I u}\right)+C^{C} \sum_{w=1}^{N_{R}} P_{r, t, m}^{R C} \\
& \left.\left.+\sum_{g t=1}^{N_{G}}\left(C_{G}^{U} Q_{g t, t, m}^{U}-C_{G}^{D} Q_{g t, t, m}^{D}\right)+C^{L S} \sum_{l=1}^{N_{L}} L_{l, t, m}^{L S}\right\}\right\}
\end{aligned}
$$

where $P_{g, t}, R_{g, t}^{U}$ and $R_{g, t}^{D}$ are power generation, spinning-up and -down reserve capacity of unit $g$ at time $t ; P_{t}^{I M}$ is imported power; $Q_{g t, t}$ is gas supply from terminal $g t ; Q_{g s, t}^{I}$ and $Q_{g s, t}^{O}$ are injection and withdrawal flow rates of gas storage $g s ; \pi_{m}$ is the probability of scenario $m ; r_{g, t, m}^{U}$ and $r_{g, t, m}^{D}$ are spinning-up and -down reserve activation for power units; $Q_{g t, t, m}^{U}$ and $Q_{g t, t, m}^{D}$ are up and down regulation of gas supply; $Q_{g s, t, m}^{O u}$ and $Q_{g s, t, m}^{I u}$ are regulation of gas storage; $P_{r, t, m}^{R C}$ and $L_{l, t, m}^{L,}$ are curtailment of wind/PV and load. $N_{E}, N_{G}, N_{G S}$, $N_{R}, N_{L}$ and $N_{M}$ are number of thermal generators, gas terminals, gas storage, wind/PV plants, loads and scenarios. The coefficient $C$ is cost per unit. Reserve cost $C_{g}^{C U}$ and $C_{g}^{C D}$ were set higher than power generation cost $C_{g}^{E}$. Reserve activation cost $C_{g}^{U}$ and $C_{g}^{D}$ were set as same as $C_{g}^{E}$. The activation of downward reserve of power or down regulation of gas supply implies cost saving. However, regulation of gas storage capacity always induces more operation cost.

\section{B. First-stage constraints}

Binary variables $u, y$ and $z$ are defined in (7) to indicate the on/off states, start-up and shut-down of units. (8)-(10) are limits for power generation and ramps [11]. (11) limits the up and down reserve capacity. The state-of-charge of electricity storage is determined by (12). (13) imposes maximum limits of charging and discharging for battery storage. (14) limits the schedule of wind and PV power below their day-ahead forecasts. (15)-(18) are constraints of gas supply and storage. (19) is gas consumed by power generation. (20) and (21) are balance equations for electricity and gas. The constraints of min-up/down time for generators can be found in [24]

$$
\begin{aligned}
& y_{g, t}-z_{g, t}=u_{g, t}-u_{g, t-1}, y_{g, t}+z_{g, t} \leq 1 \\
& u_{g, t} \underline{P}_{g} \leq P_{g, t} \leq u_{g, t} \bar{P}_{g} \\
& P_{g, t}-P_{g, t-1} \leq R U_{g}\left(1-y_{g, t}\right)+S U_{g} y_{g, t} \\
& P_{g, t-1}-P_{g, t} \leq R D_{g}\left(1-z_{g, t}\right)+S D_{g} z_{g, t} \\
& R_{g, t}^{U} \leq \bar{P}_{g}-P_{g, t}, R_{g, t}^{D} \leq P_{g, t}-u_{g, t} \underline{P}_{g}
\end{aligned}
$$

$$
\begin{aligned}
& S_{q, t}^{E S}=S_{q, t-1}^{E S}+P_{q, t}^{E C} \eta_{c}-P_{q, t}^{E D} / \eta_{d} \\
& 0 \leq P_{q, t}^{E C} \leq \bar{P}_{q}^{C}, 0 \leq P_{q, t}^{E D} \leq \bar{P}_{q}^{D} \\
& 0 \leq P_{r, t}^{R} \leq P_{r, t}^{R A} \\
& 0 \leq Q_{g t, t} \leq \bar{Q}_{g t} \\
& \underline{S}_{g s} \leq S_{g s, t}^{\text {total }} \leq \bar{S}_{g s} \\
& S_{g s, t}^{\text {total }}=S_{g, t+t-1}^{\text {total }}+Q_{g s, t}^{I}-Q_{g s, t}^{O} \\
& 0 \leq Q_{g s, t}^{I} \leq \bar{Q}_{g s}^{I}, 0 \leq Q_{g s, t}^{O} \leq \bar{Q}_{g s}^{O} \\
& P_{g, t}=\phi H Q_{g, t}^{E} \\
& \sum_{g=1}^{N_{E}} P_{g, t}+\sum_{r=1}^{N_{R}} P_{r, t}^{R}+P_{t}^{I M}+P_{r e}= \\
& \sum_{l=1}^{N_{L}} L_{l, t}+\sum_{q=1}^{N_{E S}} P_{q, t}^{E C}-\sum_{q=1}^{N_{E S}} P_{q, t}^{E D} \\
& \sum_{g t=1}^{N_{G}} Q_{g t, t}+\sum_{g s=1}^{N_{S}}\left(Q_{g s, t}^{O}-Q_{g s, t}^{I}\right)= \\
& \sum_{g=1}^{N_{H}}\left(Q_{g, t}^{E}+Q_{g}^{S U} y_{g, t}+Q_{g}^{S D} z_{g, t}\right)+\sum_{d=1}^{N_{D}} Q_{d, t}^{D}
\end{aligned}
$$

where $\underline{P}_{g}$ and $\bar{P}_{g}$ are lower and upper limits of power generation units; $R U_{g}$ and $R D_{g}$ are ramp rates; $S U_{g}$ and $S D_{g}$ are start-up and shut-down rates; $S_{q, t}^{E S}, P_{q, t}^{E C}$ and $P_{q, t}^{E D}$ are state of charge (SOC), charging and discharging power of electricity storage $q ; \eta_{c}$ and $\eta_{d}$ are efficiency of charging and discharging; $\bar{P}_{q}^{C}$ and $\bar{P}_{q}^{D}$ are limits for charging and discharging; $P_{r, t}^{R}$ and $P_{r, t}^{R A}$ are day-ahead schedule and point forecast of wind/PV power; $\bar{Q}_{g t}$ is upper limit of gas supply from terminal $g t ; S_{g s, t}^{t o t a l}, \underline{S}_{g s}$ and $\bar{S}_{g s}$ are working capacity of gas storage and its limits; $Q_{g s, t}^{I}$ and $Q_{g s, t}^{O}$ are injection and withdrawal rates of gas storage; $\underline{Q}_{g s}^{I}$ and $\bar{Q}_{g s}^{O}$ are upper limits for injection and withdrawal rates of gas storage; $\phi$ and $H$ are thermal efficiency and heating value of gas turbines; $Q_{g, t}^{E}$ is gas consumption of power generation; $P_{r e}$ is renewable power generation except wind and $\mathrm{PV} ; L_{l, t}$ and $Q_{d, t}^{D}$ are electrical load and gas demand; $Q_{g}^{S U}$ and $Q_{g}^{S D}$ are gas consumption by start-up and shut-down of thermal units.

\section{Second-stage constraints}

(22) sets limits for reserve activation. (23) defines load shifting and load shedding. (24) and (25) are constraints of electricity storage. (26) limits wind/PV curtailment. (27)-(29) are regulations of gas supply and gas storage. (30) is increased gas consumption by power generation. (31) and (32) are balance equations for electricity and gas.

$$
\begin{aligned}
& r_{g, t, m}^{U} \leq R_{g, t}^{U}, r_{g, t, m}^{D} \leq R_{g, t}^{D} \\
& \sum_{t=1}^{T} L_{l, t}=\sum_{t=1}^{T} L_{l, t, m}^{D R}, 0 \leq L_{l, t, m}^{L S} \leq L_{l, t, m}^{D R} \\
& \tilde{S}_{q, t, m}=S_{q, t}^{E S}+P_{q, t, m}^{C U} \eta_{c}-P_{q, t, m}^{D U} / \eta_{d} \\
& 0 \leq P_{q, t, m}^{C U} \leq \bar{P}_{q}^{C}-P_{q, t}^{E C}, 0 \leq P_{q, t, m}^{D U} \leq \bar{P}_{q}^{D}-P_{q, t}^{E D} \\
& P_{r, t, m}^{R C} \leq P_{r, t, m}^{R S} \\
& 0 \leq Q_{g t, t, m}^{U} \leq \bar{Q}_{g t}-Q_{g t, t}, 0 \leq Q_{g t, t, m}^{D} \leq Q_{g t, t} \\
& \tilde{S}_{g s, t, m}^{t o t a}=S_{g s, t}^{t o t a l}+Q_{g s, t, m}^{I u}-Q_{g s, t, m}^{O u} \\
& 0 \leq Q_{g s, t, m}^{I u} \leq \bar{Q}_{g s}^{I}-Q_{g s, t}^{I}, 0 \leq Q_{g s, t, m}^{O u} \leq \bar{Q}_{g s}^{O}-Q_{g s, t}^{O} \\
& r_{g, t, m}^{U}-r_{g, t, m}^{D}=\phi H \hat{Q}_{g, t, m}^{E} \\
& \sum_{r=1}^{N_{R}}\left(P_{r, t, m}^{R S}-P_{w, t}^{R}-P_{r, t, m}^{R C}\right)+\sum_{g=1}^{N_{E}}\left(r_{g, t, m}^{U}-r_{g, t, m}^{D}\right) \\
& +\sum_{q=1}^{N_{E S} S}\left(P_{q, t, m}^{D U}-P_{q, t, m}^{C U}\right)=\sum_{l=1}^{N_{L}}\left(L_{l, t, m}^{D R}-L_{l, t}-L_{l, t, m}^{L S}\right) \\
& \sum_{g t=1}^{N_{G}}\left(Q_{g t, t, m}^{U}-Q_{g, t, m}^{D}\right)+\sum_{g s=1}^{N_{G S}}\left(Q_{g s, t, m}^{O u}-Q_{g s, t, m}^{I u}\right)=\sum_{g=1}^{N_{E}} \hat{Q}_{g, t, m}^{E}
\end{aligned}
$$


where $L_{l, t, m}^{D R}$ is shifted electrical load; $\tilde{S}_{q, t, m}$ is second-stage SOC of electricity storage; $P_{q, t, m}^{C U}$ and $P_{q, t, m}^{D U}$ are up regulation of charging and discharging power; $P_{r, t, m}^{R C}$ is curtailment of wind/PV; $P_{r, t, m}^{R S}$ is the $m$ th forecast scenario of wind/PV $r ; \tilde{S}_{g s, t, m}^{t o t a l}$ is second-stage working capacity of gas storage; $\hat{Q}_{g, t, m}^{E}$ is the increment of gas consumed by power generation.

\section{Case Study}

\section{A. Input data and assumptions}

The studied system is depicted in Fig. 2. Under the Community Renewables 2030 scenario [1], the total power generation capacity is $153.4 \mathrm{GW}$. The wind capacity at transmission and distribution levels are $42.1 \mathrm{GW}$ and $11.2 \mathrm{GW}$, and the PV capacity at distribution and building levels are $20 \mathrm{GW}$ and $9.5 \mathrm{GW}$. The capacity of various generating technologies are: gas-fired $22.5 \mathrm{GW}$, nuclear $4.6 \mathrm{GW}$, hydro $2.1 \mathrm{GW}$, electricity storage $12.9 \mathrm{GW}$, inter-connectors $16.5 \mathrm{GW}$ and other renewable generation $12 \mathrm{GW}$. The capacity of each gas-fired generator is 500MW. The nuclear and hydro are must-run generators. Renewable generation except wind/PV were assumed to be fixed according to an capacity factor. The peak electric demand is $57.4 \mathrm{GW}$. The annual gas demand is $55 \mathrm{bcm}$ and supplied by four gas terminals with maximum capacity of $90 \mathrm{mcm} /$ day each. The capacity of two gas storage facilities are $34 \mathrm{mcm}$ and $37.3 \mathrm{mcm}$. They were used to simulate the transmission line-pack and short-term gas storage at distribution level. Other parameters can be found in [14] and [25].
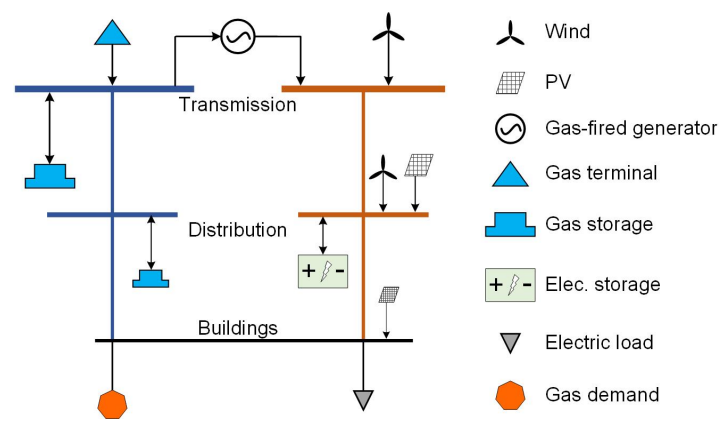

Fig. 2. The representation of the studied multi-scale energy system

\section{B. Cases}

Stochastic dispatching under different uncertainty models was studied in three cases with different flexibility options:

- GasOnly: Gas-fired generators and gas storage.

- GasStor: GasOnly with electricity storage.

- GasStorDR: GasStor with 10\% shiftable electrical load .

A deterministic model (DM) was also considered as a benchmark. The one-stage DM assumes the forecasts are perfect without uncertainty and no reserve capacity is needed.

\section{Results and discussions}

Fig. 3 shows average daily operation cost over a year for different cases and different models. The GasStorDR with combined electricity storage and demand response (DR) has the lowest cost. By using electricity storage on GasOnly, the cost is significantly reduced. However, allowing $10 \%$ shiftable demand can only bring a very small cost reduction. By comparing the breakdowns of the total costs of GasOnly and GasStor, it can be seen that the cost reduction is mainly due to the reduction of reserve (for uncertainty models only), power import and wind/PV curtailment.

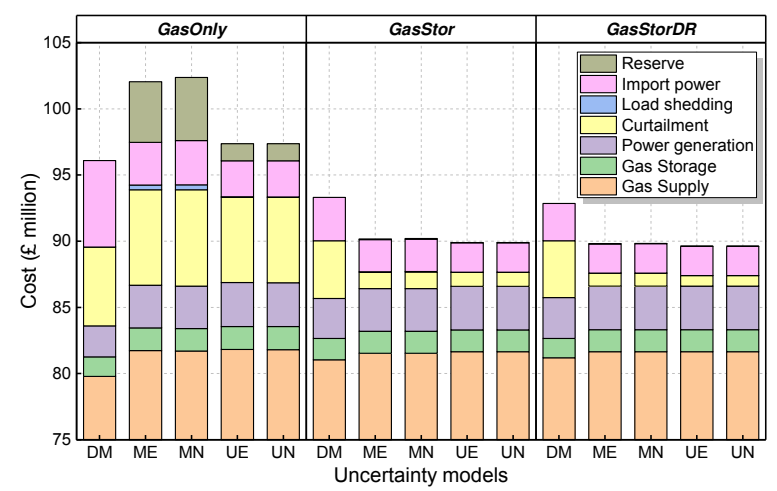

Fig. 3. Average daily system operation cost

By comparing the DM with uncertainty models, it is shown that the DM costs less for GasOnly. This is mainly because the DM assumes no reserve, but obviously it would put the system at considerable risk in practice. Also note that the DM consumes less gas for power generation but needs more expensive power import, as the CCGTs and gas storage are not flexible enough to meet the increased power requirement. For GasStor and GasOnly, where the system is provided with more flexibility, the cost of uncertainty models becomes lower than the DM, as the DM has much higher renewable curtailment.

Regarding different uncertainty models, the cost for multivariate models are generally higher than that for univariate models. The difference is much more significant for the case GasOnly which is less flexible than GasStor and GasStorDR. By analysing the forecast scenarios, it is found that the scenarios generated by multivariate models are more diverse and conforming to the temporal characteristics of practical time series, while the scenarios generated by univariate models are random but very close to each other. This reveals that the univariate models could underestimate the uncertainty in the system and thus lead to lower cost. No notable difference is observed between empirical and normal distribution models.

The daily reserve cost is given in Fig. 4. It is shown that a large proportion of the total reserve cost is caused by reserve capacity which is to compensate uncertainties at the second stage. Note that the reserve activation cost is positive when more upward reserve than downward reserve is activated, otherwise it is negative. For the GasOnly the system needs higher upward reserve for multivariate models and needs higher downward reserve for univariate models. For GasStor and GasStorDR, the system always requires more downward reserve and there is no significant difference between different uncertainty models.

Fig. 5 shows the state of charge (SOC) of electricity storage at the first stage for GasStor. The storage operation for 


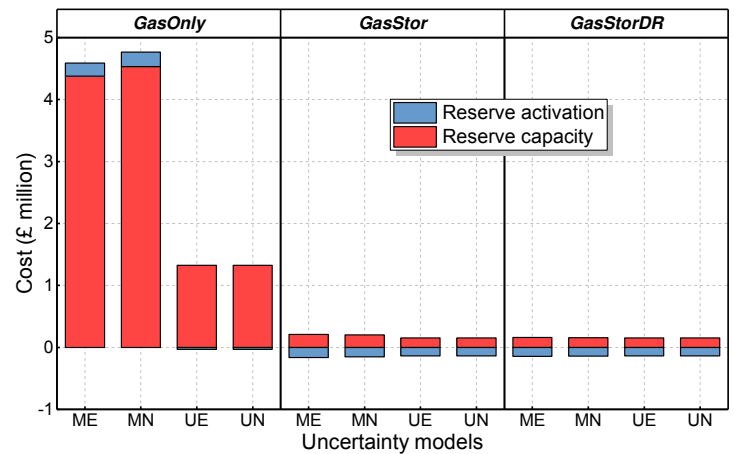

Fig. 4. Average daily reserve cost

multivariate models is more conservative than univariate ones, because the multivariate models represent higher uncertainty which makes the storage keep more regulation capacity to compensate possible large deviations at second stage.

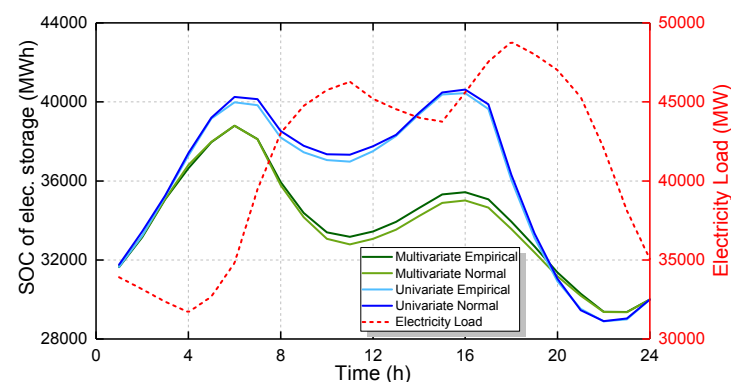

Fig. 5. Load profile and first-stage SOC of eletricity storage for GasStor

\section{CONCLUSION AND FUTURE WORK}

It was demonstrated that flexibility technologies are crucial to ensure a low-carbon, economic and secure GB energy system for the future. In the studies, along with the gas supply system, electricity storage is the most effective option to reduce operation cost, especially in terms of reduced reserve, power import and renewable curtailment. However, additional $10 \%$ demand shifting has little impact at whole-system level.

The significance of uncertainty characterisation depends on the level of system flexibility. A highly flexible system will be insensitive to different uncertainty models, and thus can avert the risks caused by inaccurate characterisation of uncertainty.

The proposed framework could be readily applied to study other integrated energy systems, by adjusting parameters, adopting different flexibility options and modifying energy system model (e.g., considering detailed energy network constraints). The results will allow understanding where and how much flexibility will be needed in stochastic energy systems, and how these needs will best be met by a 'flexibility mix' of technologies.

\section{REFERENCES}

[1] "Net Zero-The UK's contribution to stopping global warming," Committee on Climate Change, London, UK, May 2019.

[2] "Future Energy Scenarios 2019," National Grid Electricity System Operator, London, UK, July 2019.
[3] M. Qadrdan, H. Ameli, G. Strbac, and N. Jenkins, "Efficacy of options to address balancing challenges: Integrated gas and electricity perspectives," Appl. Energy, vol. 190, pp. 181-190, Mar. 2017.

[4] A. Orths et al., "Flexibility from energy systems integration: supporting synergies among sectors," IEEE Power Energy Mag., vol. 17, no. 6, pp. 67-78, Nov.-Dec. 2019.

[5] P. D. Lund, J. Lindgren, J. Mikkola, and J. Salpakari, "Review of energy system flexibility measures to enable high levels of variable renewable electricity," Renew. Sust. Energ. Rev., vol. 45, pp. 785-807, May 2015.

[6] C. M. Correa-Posada, P. Sánchez-Martín and S. Lumbreras, "Securityconstrained model for integrated power and natural-gas system,'Journal of Modern Power Systems and Clean Energy, vol. 5, no. 3, pp. 326-336, May 2017.

[7] B. Zhao, A. J. Conejo and R. Sioshansi, "Using Electrical Energy Storage to Mitigate Natural Gas-Supply Shortages," IEEE Transactions on Power Systems, vol. 33, no. 6, pp. 7076-7086, Nov. 2018.

[8] X. Zhang, M. Shahidehpour, A. Alabdulwahab and A. Abusorrah, "Hourly Electricity Demand Response in the Stochastic Day-Ahead Scheduling of Coordinated Electricity and Natural Gas Networks," IEEE Transactions on Power Systems, vol. 31, no. 1, pp. 592-601, Jan. 2016

[9] X. Yue, S. Pye, J. DeCarolis, F. Li, F. Rogan, and B. Gallachoir, "A review of approaches to uncertainty assessment in energy system optimization models," Energy Strateg Rev, vol. 21, pp. 204-217, Aug. 2018.

[10] C. Ordoudis, P. Pinson, and J. Morales, "An Integrated Market for Electricity and Natural Gas Systems with Stochastic Power Producers," Eur J Oper Res, vol. 272, no. 2, pp. 642-654, Jan 162019.

[11] M. Yan, N. Zhang, X. Ai, M. Shahidehpour, C. Kang, and J. Wen, "Robust two-stage regional-district scheduling of multi-carrier energy systems with a large penetration of wind power," IEEE Trans. Sustain. Energy, vol. 10, no. 3, pp. 1227-1239, Jul. 2019.

[12] E. Lannoye, D. Flynn, and M. O'Malley, "Evaluation of power system flexibility," IEEE Trans. Power Syst., vol. 27, no. 2, pp. 922-931, May 2012.

[13] E. Lannoye, D. Flynn, and M. O'Malley, "Transmission, variable generation, and power system flexibility," IEEE Trans. Power Syst., vol. 30, no. 1, pp. 57-66, Jan. 2015.

[14] M. Qadrdan, M. Chaudry, J. Wu, N. Jenkins, and J. Ekanayake, "Impact of a large penetration of wind generation on the GB gas network," Energy Policy, vol. 38, no. 10, pp. 5684-5695, Oct. 2010.

[15] M. Zeyringer, J. Price, B. Fais, P. H. Li, and E. Sharp, "Designing lowcarbon power systems for Great Britain in 2050 that are robust to the spatiotemporal and inter-annual variability of weather," Nat. Energy, vol. 3, no. 5, pp. 395-403, May 2018.

[16] P. Pinson, "Very-short-term probabilistic forecasting of wind power with generalized logit-normal distributions," Journal of the Royal Statistical Society Series C-Applied Statistics, vol. 61, pp. 555-576, 2012.

[17] D. Hung, N. Mithulananthan, and K. Lee, "Determining PV penetration for distribution systems with time-varying load models," IEEE Trans. Power Syst., vol. 29, no. 6, pp. 3048-3057, Nov. 2014.

[18] B. Alonzo, H. Ringkjob, B. Jourdier, P. Drobinski, R. Plougonven, and P. Tankov, "Modelling the variability of the wind energy resource on monthly and seasonal timescales," Renew. Energy, vol. 113, pp. 1434 1446, Dec. 2017.

[19] P. Bett and H. Thornton, "The climatological relationships between wind and solar energy supply in Britain," Renew. Energy, vol. 87, pp. 96-110, Mar 2016.

[20] A. Schellenberg, W. Rosehart, and J. Aguado, "Cumulant-based probabilistic optimal power flow (P-OPF) with Gaussian and Gamma distributions," IEEE Trans. Power Syst., vol. 20, no. 2, pp. 773-781, May 2005.

[21] Y. Zhao, L. Ye, Z. Li, X. Song, Y. Lang, and J. Su, "A novel bidirectional mechanism based on time series model for wind power forecasting," Appl. Energy, vol. 177, pp. 793-803, Sep. 2016.

[22] X. Ma, Y. Sun, and H. Fang, "Scenario Generation of Wind Power Based on Statistical Uncertainty and Variability," IEEE Trans. Sustain. Energy, vol. 4, no. 4, pp. 894-904, Oct. 2013.

[23] J. Morales, A. Conejo, H. Madsen, P. Pinson, and M. Zugno, Integrating renewables in electricity markets: operational problems. New York: Springer, 2013.

[24] J. Arroyo and A. Conejo, "Optimal response of a thermal unit to an electricity spot market," IEEE Trans. Power Syst., vol. 15, no. 3, pp. 1098-1104, Aug. 2000.

[25] M. Joos and I. Staffell, "Short-term integration costs of variable renewable energy: wind curtailment and balancing in Britain and Germany," Renew. Sust. Energ. Rev., vol. 86, pp. 45-65, Apr. 2018. 